\title{
Idiopathic Neonatal Subpial Hemorrhage with Underlying Cerebral Infarct: Imaging Features and Clinical Outcome
}

\author{
(D) Z. Assis, (D) A. Kirton, (D) A. Pauranik, (D) M. Sherriff, and (D) X.-C. Wei
}

\begin{abstract}
BACKGROUND AND PURPOSE: Neonatal subpial hemorrhage with underlying cerebral infarct is a previously described but poorly understood clinicoradiographic syndrome. We sought to further characterize the cranial ultrasound and MR imaging characteristics and associated outcomes of this condition across the full range of gestational ages, including extreme and very preterm neonates.

MATERIALS AND METHODS: This was a single tertiary pediatric center retrospective case series. Brain MR imaging and cranial ultrasound of neonates with subpial hemorrhage with underlying cerebral infarct were identified from a population-based radiology registry (2006-2020). Original images were reviewed by 2 neuroradiologists blinded to history and outcome. Clinical presentation, course, and outcome at $>12$ months were abstracted from medical records. The diagnostic utility of cranial ultrasound was compared with that of MR imaging.

RESULTS: Sixteen patients were included (median gestational age, 36.5 weeks; range, 27-41 weeks; 31\% premature). MR images were obtained acutely at the time of presentation between days 0 and 9 of life. On T2WI and DWI, a consistent presence of a hypointense subpial bleed and an underlying hyperintense cerebral cortex were recognized, which created a distinct MR imaging pattern resembling the yin-yang symbol. Findings of all the MRAs and MRVs were normal. Cranial ultrasound detected 6 of 7 MR imaging lesions with sonographic features correlating well with MR imaging. The 3 extreme or very preterm neonates did not survive. The remainder survived with relatively mild neurologic deficits.
\end{abstract}

CONCLUSIONS: Subpial hemorrhage with underlying infarction is a recognizable condition with unique MR imaging and sonographic features. Improved recognition may advance understanding of risk factors and outcomes.

ABBREVIATION: GRE $=$ gradient recalled-echo

$\mathrm{M}$ ost children with perinatal stroke experience life-long neurologic disabilities. ${ }^{1,2}$ Among the many subtypes of perinatal stroke, there is increasing awareness of neonatal subpial hemorrhage with underlying cerebral infarction diagnosed in the neonatal period. ${ }^{3}$ This entity was first described by Huang and Robertson. ${ }^{4}$ In their report of 7 term neonates who had spontaneous superficial parenchymal and leptomeningeal hemorrhage on CT and MR imaging, 4 had short-term clinical follow-up and were neurologically normal. Recently, Cain et $\mathrm{al}^{5}$

Received June 26, 2020; accepted after revision August 19.

From the Departments of Radiology (Z.A., A.K., A.P., X.-C.W.), Clinical

Neurosciences and Pediatrics (A.K.), University of Calgary, Calgary, Alberta, Canada; and Department of Diagnostic Imaging (Z.A., A.P., M.S., X.-C.W.), Alberta Children's Hospital, Calgary, Alberta, Canada.

This study was previously presented, in part, at: Annual Meeting of the American Society of Pediatric Neuroradiology, January 18-20, 2019; New Orleans, Louisiana.

Please address correspondence to Xing-Chang Wei, MB, MSc, FRCPC, Diagnostic Imaging, Alberta Children's Hospital, 28 Oki Dr NW, Calgary, AB T3B 6A8, Canada; e-mail:weix@ucalgary.ca

http://dx.doi.org/10.3174/ajnr.A6872

reported 17 neonates who had subpial hemorrhage with underlying intraparenchymal cytotoxic edema on MR imaging shortly after birth. All of these term or late-preterm neonates survived except for one who succumbed to complications of congenital heart disease.

With such rare descriptions, the incidence, pathogenesis, possible risk factors, and outcomes of this clinicoradiographic syndrome are unknown. The condition has also been inconsistently named, contributing to under-recognition and hindering studies of pathogenesis, management, and outcome. To our knowledge, occurrence in early preterm neonates has not been reported. Moreover, the sonographic features and diagnostic value of cranial ultrasound have not been described despite this technique often being the first-line in critically ill and preterm neonates.

In this study, we report the MR imaging and cranial ultrasound features of 16 neonates, including preterm infants, with imaging features suggestive of subpial hemorrhage with underlying brain parenchymal hemorrhagic infarction. 
Table 1: Patient demographics, clinical presentation, and outcomes

\begin{tabular}{|c|c|c|}
\hline Clinical Parameter & Subgroups & Value \\
\hline Maternal age & Mean age in years & $33.5(27-39)$ \\
\hline \multirow[t]{4}{*}{ Gestational age at birth (weeks) } & Term ( $\geq 37)$ & $11 / 16$ \\
\hline & Moderate-to-late preterm (32-37) & $2 / 16$ \\
\hline & Very preterm (28-32) & $2 / 16$ \\
\hline & Extremely preterm $(\leq 28)$ & $1 / 16$ \\
\hline \multirow[t]{2}{*}{ Sex } & Male & $5 / 16$ \\
\hline & Female & $11 / 16$ \\
\hline \multirow[t]{2}{*}{ Mode of delivery } & Vaginal & $13 / 16$ \\
\hline & Cesarean delivery & $3 / 16$ \\
\hline \multirow[t]{3}{*}{ Birth weight } & $<2.5 \mathrm{~kg}$ & $5 / 16$ \\
\hline & $>2.5 \mathrm{~kg}$ & $11 / 16$ \\
\hline & Mean & $2.2 \mathrm{~kg}$ \\
\hline \multirow[t]{2}{*}{ Birth assistance in vaginal delivery } & No assistance & $10 / 13$ \\
\hline & Vacuum assistance & $3 / 13$ \\
\hline \multirow{3}{*}{ Clinical presentation } & Apnea & $10 / 16$ \\
\hline & Seizure & $9 / 16$ \\
\hline & Encephalopathy & $3 / 16$ \\
\hline APGAR score at $1 \mathrm{~min}$ & & 6.3 (range, 1-9) \\
\hline APGAR score at $5 \mathrm{~min}$ & & 8.4 (range, 4-9) \\
\hline Age at onset of symptoms & & 2.1 days (range, $1-6$ days of life) \\
\hline Age at most recent follow-up & & 18.8 months (range, $1-90$ months) \\
\hline \multirow[t]{2}{*}{ Abnormal coagulation/hematology profile } & Maternal & $0 / 16$ \\
\hline & Neonatal & 1/16 (elevated hematocrit) \\
\hline \multirow[t]{3}{*}{ Outcome } & Death & $3 / 16$ (1 extremely preterm, 2 very preterm) \\
\hline & With neurologic deficits & $2 / 16$ \\
\hline & No neurologic deficits & $11 / 16$ \\
\hline
\end{tabular}

\section{MATERIALS AND METHODS Population}

The participants were retrospectively identified by a pediatric neuroradiologist through a neuroimaging teaching case data base stored on the institutional (Alberta Children's Hospital, Calgary) computer system. The terms "hemorrhage" and "neonate" were searched in the data base. Clinical images of the returned subjects were retrospectively reviewed on the clinical PACS, from which subjects who had subpial hemorrhage were included in this study. Information of patient demographics, clinical course, and laboratory investigation was collected from the electronic medical chart. The study was approved by the institutional research ethics board.

\section{Image Acquisition}

MR images of the brain were obtained for clinical reasons on different clinical scanners in 3 different hospitals (Alberta Children's Hospital, Foothills Medical Centre and South Health Campus, all located in Calgary city) in the city from April 2006 to April 2020. Scanners were $1.5 \mathrm{~T}$ or $3 \mathrm{~T}$ in field strength. The MR imaging and specific scanning parameters varied, but the same essential anatomic sequences used in the current study were always performed, including sagittal and axial T1WI, axial and coronal FSE T2WI, axial DWI (and coronal in some cases), and axial gradient recalledecho (GRE) T2*-weighted imaging. Coronal DWI was performed in all initial scans and in most of the follow-up scans. SWI was performed in some cases instead of GRE T2*WI. TOF-MRA and noncontrast MRV, including 2D-TOF-MRV and 3D phase-contrast MRV, were performed in most cases (see RESULTS).

Cranial ultrasound studies were performed on a variety of machines in multiple centers during the same timeframe. Standard oblique sagittal and oblique coronal still images and cine loops were obtained through the anterior fontanelle in all cases.

\section{Image Analysis}

Analysis was performed by 2 pediatric neuroradiologists (Z.A., X.-C.W.), who were blinded to clinical presentation and outcome. Clinical images were retrospectively reviewed on the clinical PACS. The presence, location, size, and signal intensity of subpial hemorrhage and the underlying brain parenchyma were recorded on a standardized data-capture form. All available MRA and MRV images were reviewed. For those participants with multiple MR imaging and sonographic scans, the initial scans were analyzed to assess the diagnostic performance of MR imaging and ultrasound. Follow-up MR images were analyzed to assess the natural course of the disease. In addition, the MRA images from later scans were reviewed to exclude vascular malformations that may not have been detected in earlier scans. Cranial ultrasound findings were compared with brain MR imaging findings.

\section{RESULTS}

\section{Population}

Sixteen patients were included in the study. Patient demographics are summarized in Table 1 . Most $(n=11)$ were born at term gestation ( $\geq 37$ weeks), 2 were moderate-to-late preterm (32-37 weeks), 2 were very preterm (28-32 weeks), and 1 was extremely preterm ( $\leq 28$ weeks). Of note, almost $70 \%$ of the infants were born by vaginal delivery.

\section{Clinical Presentation, Investigations, and Outcome}

Clinical presentation and outcome are also summarized in Table 1. Perinatal histories were generally unremarkable. No maternal 

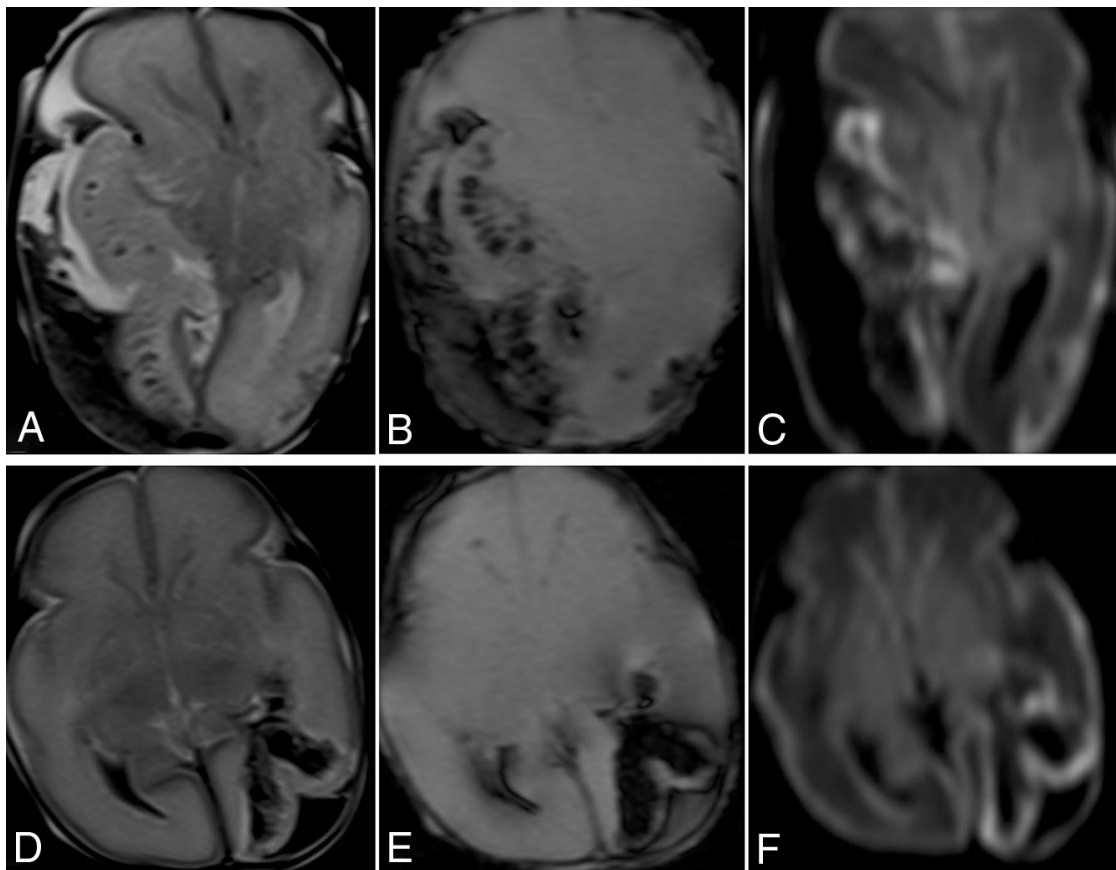

T2WI

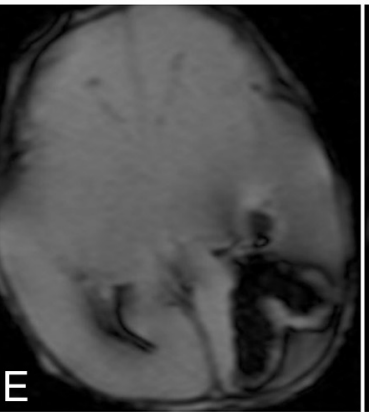

GRE T2*WI

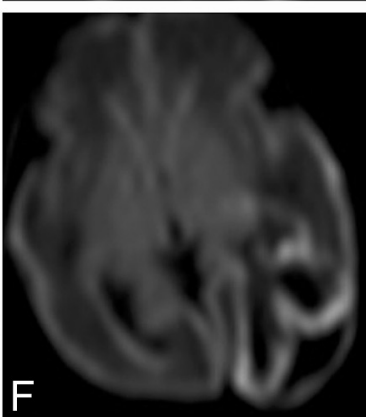

DWI

FIG 1. MR imaging of 2 preterm neonates who did not survive to discharge from the neonatal unit. The first patient $(A-C)$ was born at 27 weeks 3 days' gestation and had a large subpial hemorrhage and a large underlying hemorrhagic infarct. The second patient $(D-F)$ was born at 28 weeks 1 day of gestation. She had a relatively small subpial hemorrhage and underlying hemorrhagic infarct but had engorged deep medullary veins in the bilateral cerebral hemispheres that may have had small thrombosis (not shown).

history of smoking or substance abuse was found. Three patients had a history of controlled gestational hypertension. APGAR scores were available for 12 of the 16 neonates ( 9 term, 3 preterm) and were usually normal. Of note, 3 of the 16 patients had a clinical presentation of encephalopathy, all of whom were preterm. None of them were diagnosed with other neurologic conditions such as hypoxic-ischemic encephalopathy. Symptoms were seen as early as 4 hours after birth to 6 days of life.

Eight patients were investigated with electroencephalography, of which 7 showed abnormalities in the form of focal epileptiform discharges and 1 was unremarkable. Six of the 7 electroencephalograpies with abnormal findings were lateralized to the side of the subpial hemorrhage. In 2 of these 6 patients, the seizure origin on electroencephalography was consistent with the location of hemorrhage and infarction, while in the other 4 patients, the seizure origin could not be further localized. Coagulation profiles, including complete blood count, partial thromboplastin time, and international normalized ratio, were completed in all patients and were unremarkable. One patient had prenatal maternal group B streptococcal culture, for which antibiotic treatment was given, but the child was negative with no sepsis or meningitis. All neonates had at least a partial septic work-up, all of which were negative for sepsis (systemic infection).

All patients received standardized neurocritical care. Seizures were managed acutely with antiseizure medications, including levetiracetam, phenobarbital, and phenytoin. One preterm patient underwent a decompression craniectomy on day 4 of life to relieve the mass effect associated with a large hemorrhage (Fig $1 A-C$ ).

All 3 patients born extremely preterm or very preterm did not survive to discharge. One had a prenatal diagnosis of trisomy 21, severe oligohydramnios, and microcephaly on prenatal ultrasound and succumbed to severe renal failure. The other 2 patients did not have major diseases other than the intracranial abnormalities.

The other 13 patients survived and appeared to have good outcomes at a median of 18.8 months (range, 1-90 months). Among them, 11 patients had no residual seizures, neurologic deficits, or developmental issues on follow-up. One had recurrent seizures, which were controlled by low-dose levetiracetam. Another term neonate had occasional periodic breathing, which was managed with home oxygen. One patient had hemianopia on clinical examination at 2 months.

\section{MR Imaging at Presentation}

The results of the initial MR imaging and cranial ultrasound scans are summarized in Table 2. Seven patients had ultrasound as the first neuroimaging examination followed by MR imaging, and 8 had MR imaging only. One patient had cranial ultrasound only. The image quality of all scans was deemed satisfactory.

All lesions were unilateral and neocortical. Most (69\%) lesions were located in the temporal lobes, followed by the frontal (19\%), parietal (6\%), and occipital (6\%) lobes. No lesion was located in the posterior fossa. Two (2/16) patients had small contralateral subdural bleeds. On MR imaging, 8/15 patients showed mass effect in the form of a midline shift and/or uncal herniation. Of the 8 cases, 3 patients had only midline shift, and the remaining 5 had a combination of midline shift with uncal herniation. Some degree of regional mass effect with effacement of adjacent sulci was seen in all cases. No tonsillar herniation was observed.

On the initial MR imaging, irrespective of the location and size of the lesion, 2 key components were consistently seen on all MR images. The first was a focal subpial collection of fluid extending into the adjacent cerebral sulci associated with widening of the cerebral sulci and flattening of the underlying parenchyma. The subpial collection consistently demonstrated mildto-moderately hyperintense T1 signal, markedly hypointense T2 signal, and increased diffusivity on DWI and ADC maps, consistent with acute or subacute bleed (Fig 2).

The second component of the lesion was signal change in adjacent brain parenchyma. This included the cortex as well as subcortical and deeper white matter immediately underneath the 
Table 2: MR imaging and cranial ultrasound data and findings at presentation

\begin{tabular}{|c|c|c|}
\hline & No. & Percentage \\
\hline \multicolumn{3}{|l|}{ Imaging data } \\
\hline MR imaging and US & 7 & \\
\hline MR imaging only & 8 & \\
\hline US only & 1 & \\
\hline \multicolumn{3}{|l|}{ Age at imaging (range) (day) } \\
\hline MR imaging & $3.2(1-9)$ & \\
\hline US & $2.7(1-7)$ & \\
\hline MR imaging field strength (1.5T:3T) & & $12: 3$ \\
\hline \multicolumn{3}{|l|}{ MR imaging sequences } \\
\hline Core sequences $^{\mathrm{a}}$ & $15 / 15$ & \\
\hline GRE T2*WI & $11 / 15$ & \\
\hline SWI & $4 / 15$ & \\
\hline \multicolumn{3}{|l|}{ MRV performed } \\
\hline On initial MRI & $10 / 15$ & \\
\hline On repeat MRI within 4 days & $3 / 15$ & \\
\hline \multicolumn{3}{|l|}{ MRA performed } \\
\hline On initial MRI & $9 / 15$ & \\
\hline On repeat MRI within 6 weeks & $3 / 15$ & \\
\hline Lesion laterality (right:left) & $10: 6$ & $62 \%: 38 \%$ \\
\hline \multicolumn{3}{|l|}{ Lesion location } \\
\hline Temporal & $11 / 16$ & $69 \%$ \\
\hline Frontal & $3 / 16$ & $19 \%$ \\
\hline Parietal & $1 / 16$ & $6 \%$ \\
\hline Occipital & $1 / 16$ & $6 \%$ \\
\hline Parenchymal diffusion restriction & $15 / 15$ & $100 \%$ \\
\hline Parenchymal hemorrhage & $11 / 15$ & $50 \%$ \\
\hline Intraventricular hemorrhage & $3 / 15$ & $18 \%$ \\
\hline Midline shift & $8 / 15$ & $50 \%$ \\
\hline Yin-yang sign on MRI & $15 / 15$ & $100 \%$ \\
\hline Yin-yang sign on US & $6 / 6$ & $100 \%$ \\
\hline
\end{tabular}

Note:-US indicates ultrasound.

${ }^{a}$ The core MR images include sagittal and axial TTWI, axial and coronal FSE T2WI, and axial and coronal DWI.

subpial hemorrhage. On 4 of $15 \mathrm{MR}$ imaging scans, no intra-axial hemorrhage was present in the parenchyma. In these cases, both the cortex and white matter lesions consistently demonstrated uniformly hypointense T1 signal, hyperintense T2 signal, restricted diffusion, and isointense signal on GRE T2*WI or SWI (Fig $2 A-D$ ). On 6 of $15 \mathrm{MR}$ imaging scans, a small amount of hemorrhage was present in the subcortical and deep white matter components of the lesion. In these cases, the involved cortex consistently demonstrated hypointense T1 signal, hyperintense T2 signal, restricted diffusion, and isointense signal on GRE $\mathrm{T} 2{ }^{\star} \mathrm{WI}$, while the underlying white matter showed heterogeneous signal on T1WI and T2WI and was predominantly hypointense on DWI and GRE T2*WI (Fig $2 E-H$ ). On the remaining 5 MR images, a larger amount of hemorrhage was present in the subcortical and deep white matter. In these cases, the involved cortex consistently demonstrated hypointense T1 signal, hyperintense T2 signal, and restricted diffusion and large blooming artifacts on GRE T2*WI. The underlying white matter demonstrated heterogeneous signal on T1WI and T2WI and was predominantly hypointense on DWI and GRE T2*WI (Fig 2A-D). The signal characteristics of each group are summarized in Table 3.

On T2WI and DWI, the consistent presence of a dark, hypointense, subpial bleed and an underlying bright, hyperintense cerebral cortex created a distinct MR imaging pattern, resembling the yin-yang symbol in Chinese philosophy (Fig 3).

Two of the 3 patients who did not survive had MR imaging at days 1 and 2 of life, respectively. One of them was born at 27 weeks' gestation and had a large subpial hemorrhage and a large underlying hemorrhagic infarct (Fig $1 A-C$ ). The other patient was born at 28 weeks' gestation. She had a relatively small subpial hemorrhage and an underlying hemorrhagic infarct but also demonstrated engorged deep medullary veins bilaterally, suggestive of deep cerebral sinovenous thrombosis, though this could not be confirmed on imaging (Fig $1 D-F$ ). Both showed small amounts of intraventricular hemorrhage without evidence of germinal matrix hemorrhage.

Among the 15 patients who had MR images, 13 had MR venography completed either on their initial MR images or on a repeat scan within 4 days (Table 2). None of the MR venograms demonstrated thrombosis in the dural venous sinuses or the cerebral veins. Twelve patients had MR angiograms on either their initial MR images or the repeat scans within 6 weeks, all of which had normal findings.

\section{Cranial Ultrasound}

Seven patients had cranial ultrasound before brain MR imaging. The time interval between the ultrasound and MR imaging was an average of 13 hours (range, 5-24 hours). Cranial ultrasound detected the subpial hemorrhage with underlying infarct in 6 of 7 patients. The patient with false-negative ultrasound findings had the smallest lesion in this cohort, about half the size of the lesion of the first patient in Fig $2 A-D$. On the 6 sonographic scans positive for idiopathic subpial hemorrhage with underlying cerebral infarct, the subpial hemorrhage and the underlying parenchymal abnormality could be separated in all cases. Lesion size and shape correlated well with MR imaging on subjective review, though a quantitative analysis was not performed. The subpial hemorrhage was hypoechoic in 4 patients, mildly hyperechoic in 1 patient, and moderately hyperechoic in another. While the infarcted cerebral cortex and the underlying white matter with hemorrhagic infarct can be readily differentiated from each other on MR imaging, the 2 components could not be separated on ultrasound. Nonetheless, the combination of hypoechoic or mildly hyperechoic subpial collection of bleeds and the underlying markedly echogenic brain parenchyma gives a distinct sonographic pattern similar to the MR imaging yinyang sign (Fig 4).

One of the patients had 2 cranial ultrasound scans as the only neuroimaging studies before succumbing to disease. He was born at 30 weeks' gestation. The ultrasound findings in this patient were similar to those of the other 7 .

Among the 8 patients who underwent cranial ultrasound, only 3 had follow-up cranial ultrasound scans. One of the 3 had 14 repeat scans, one had 2, and the other had 1 . Three of the follow-up scans were obtained 1-4 days after the initial scan, which showed no obvious change in size, shape, or echogenicity of the subpial hemorrhage or the underlying brain parenchymal lesion. Three of the follow-up scans were obtained 8-11 days after the initial scan, which showed a mildly decreased size of the subpial hemorrhage and the underlying brain parenchymal lesion, while the subpial 

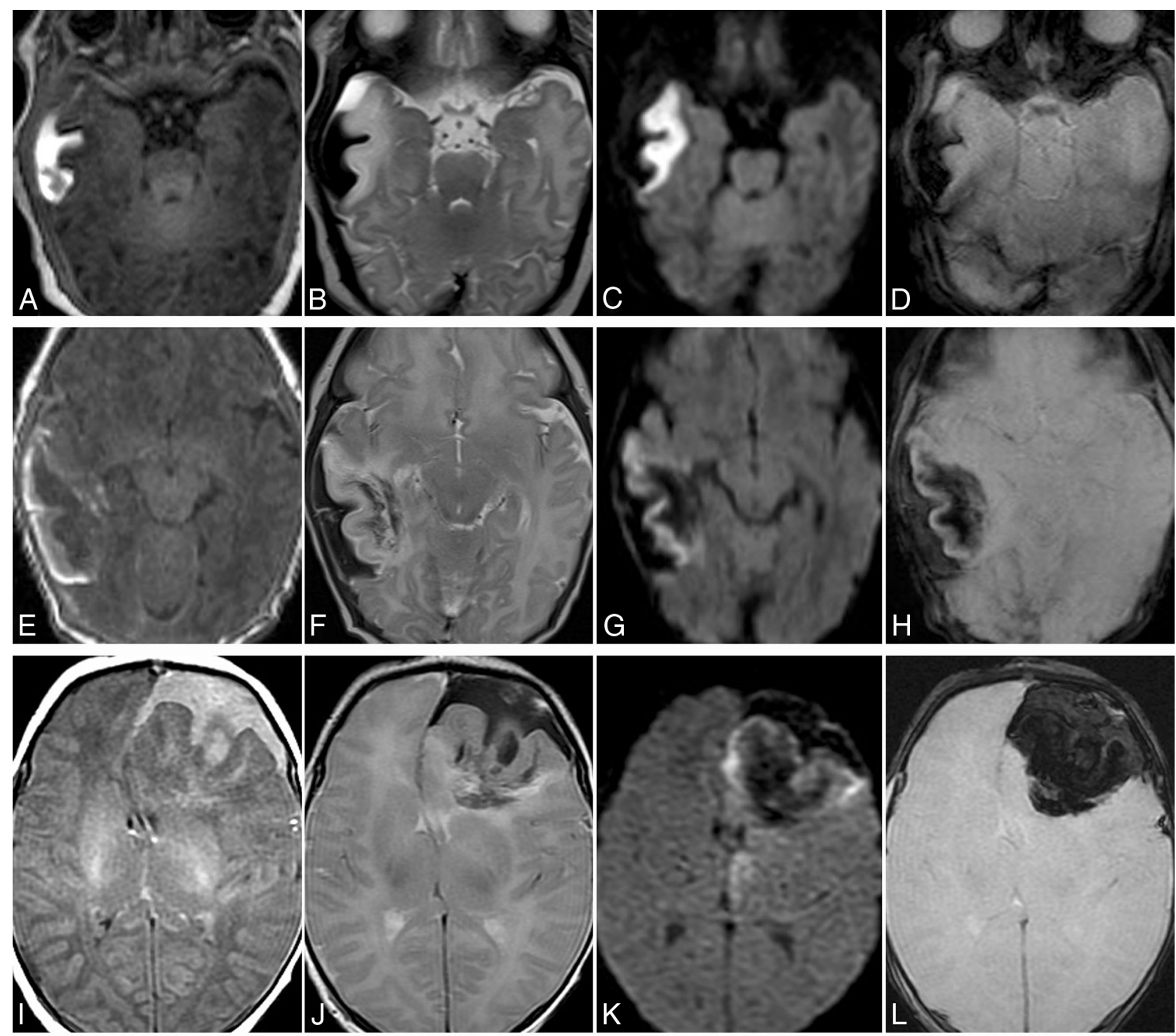

T1WI

$\mathrm{T} 2 \mathrm{WI}$

DWI

GRE T2*WI

FIG 2. MR images of 3 term neonates. The subpial hemorrhage consistently shows hyperintense $\mathrm{T} 1$ signal, hypointense $\mathrm{T} 2$ signal, no restricted diffusion, and hypointense signal on GRE T2*WI. In the first patient $(A-D)$, the underlying cerebral cortex and white matter have no hemorrhage. In the second patient $(E-H)$, a mild fan-shaped hemorrhage is seen in the underlying white matter, resulting in a hypointense signal on T2WI, $\mathrm{DWI}$, and T2*WI, while the cerebral cortex remains hyperintense on T2WI, DWI, and T2*WI. In the third patient (I-L), more severe hemorrhage is seen in the underlying white matter, leading to an obscured cerebral cortex on $\mathrm{T} 2{ }^{\star} \mathrm{WI}$ and a partially obscured cortex on DWI.

Table 3: Appearance of subpial hemorrhage and underlying brain parenchyma on initial MR imaging and US

\begin{tabular}{|c|c|c|c|c|c|}
\hline \multirow[b]{2}{*}{ Subpial Bleed, Parenchymal Lesion } & \multicolumn{4}{|c|}{ MRI } & \multirow[b]{2}{*}{ US } \\
\hline & TIWI & $\mathrm{T} 2 \mathrm{WI}$ & DWI & $\mathrm{T} 2 * \mathrm{WI}$ & \\
\hline & $\uparrow$ & $\downarrow$ & $\downarrow$ & $\downarrow$ & $\downarrow$ or $\uparrow$ \\
\hline Cortical infarct with no WM hemorrhage (4/15) & & & & & \\
\hline Cortex & $\downarrow$ & $\uparrow$ & $\uparrow$ & $\leftrightarrow$ & $\uparrow$ \\
\hline WM & $\downarrow$ & $\uparrow$ & $\uparrow$ & $\leftrightarrow$ & \\
\hline Cortical infarct with small WM hemorrhage $(6 / 15)$ & & & & & \\
\hline Cortex & $\downarrow$ & $\uparrow$ & $\uparrow$ & $\leftrightarrow$ & $\uparrow$ \\
\hline WM & Mixed & Mixed & $\downarrow$ & $\downarrow$ & \\
\hline Cortical infarct with large WM hemorrhage $(5 / 15)$ & & & & & \\
\hline Cortex & $\downarrow$ & $\uparrow$ & $\uparrow$ & $\downarrow$ & $\uparrow$ \\
\hline WM & Mixed & Mixed & & & \\
\hline
\end{tabular}

Note: $-\leftrightarrow$ indicates isointense or isoechoic; $\uparrow$, hyperintense or hyperechoic; $\downarrow$, hypointense or hypoechoic; US, ultrasound. 

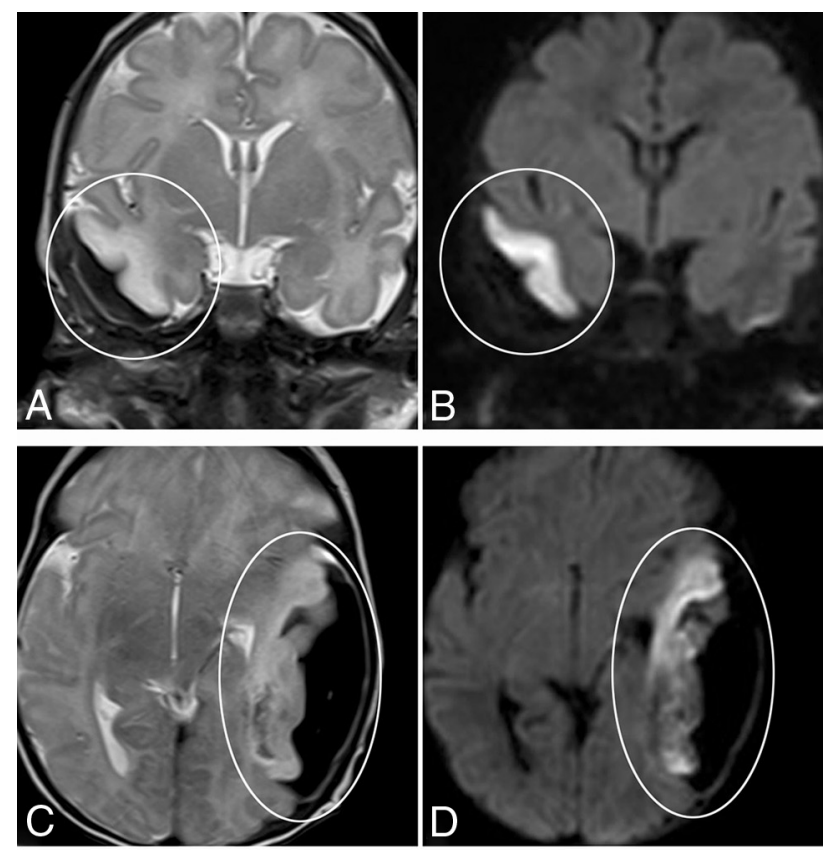

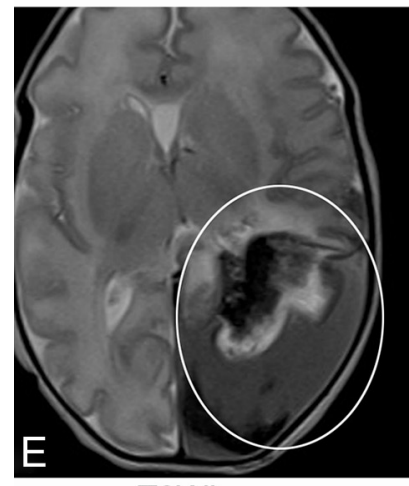

T2WI

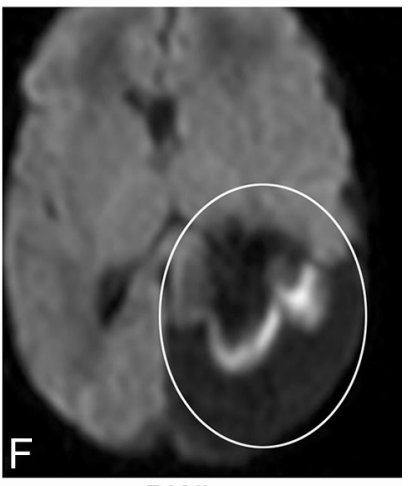

DWI

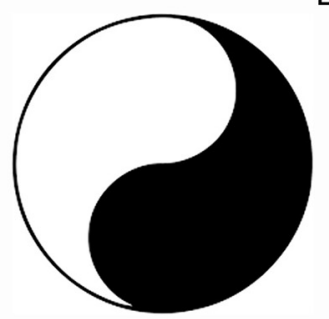

FIG 3. Yin-yang sign. $\mathrm{T} 2 \mathrm{WI}$ and $\mathrm{DWI}$ of 3 term neonates with subpial hemorrhage are shown. In the brain parenchyma underlying the subpial bleed, no hemorrhage is seen in the first patient ( $A$ and $B$ ); mild hemorrhage, in the second patient ( $C$ and $D)$; and severe hemorrhage, in the third patient ( $E$ and $F$ ). Irrespective of the presence or degree of intraparenchymal hemorrhage, the combination of a dark subpial fluid collection and a bright underlying cerebral cortex forms a consistent, distinct image pattern (circled areas in $A-F$ ), resembling the yin-yang symbol in Chinese philosophy.

hemorrhage changed from being hypoechoic to mildly echogenic and the underlying brain parenchymal lesions changed from being markedly echogenic to mildly echogenic.

\section{Follow-Up MR Imaging}

Twelve of the 15 patients had follow-up MR imaging, among whom 7 patients had 1 follow-up scan, 8 had 2, and 1 had 3 .
The MR imaging appearances of the subpial hemorrhage and underlying brain parenchyma on follow-up scans are summarized in Table 4. Of note, none of the follow-up MR images showed an interval increase in the size of the subpial hemorrhage or the underlying brain abnormality compared with the initial scans.

\section{DISCUSSION}

In 2004, Huang and Robertson ${ }^{4}$ first reported 7 term neonates with idiopathic superficial parenchymal and leptomeningeal (ie, subpial or subarachnoid) hemorrhage on MR imaging and CT. Not until 16 years later did Cain et $\mathrm{al}^{5}$ report MR images of another cohort of 17 term and late-preterm neonates with subpial hemorrhage and cytotoxic edema in the underlying brain parenchyma. Our study expands the description of this unique syndrome, reporting 16 neonates with subpial hemorrhage and underlying cerebral infarction. The contributions of this study are multifold.

First, our cohort of patients included 3 either extremely preterm ( $\leq 28$ weeks' gestation) or very preterm (28-32 weeks' gestation) neonates, all of whom did not survive to discharge. In the case series of Huang and Robertson ${ }^{4}$ and that of Cain et al, ${ }^{5}$ the patients were either term or late-preterm, and all survived with relatively minor neurologic deficits. Our sample size is small, preterm neonates are already at high risk of mortality, and the direct cause of death may not be attributable to the intracranial abnormalities. Therefore, we cannot draw any conclusions regarding possible associations between this radiographic syndrome and outcome. However, we hope these descriptions will improve recognition of this disorder by neonatologists and neurologists and alert them that extremely preterm or very preterm neonates with this condition may not have the same relatively good outcome as late-preterm or term neonates.

Second, we studied the sonographic features of idiopathic subpial hemorrhage with underlying cerebral infarction. In previous studies, only CT and MR images were analyzed and reported. However, because cranial ultrasound is often the first imaging technique in evaluating neonates with neurologic concerns due to its easy accessibility and ability to be performed at the bedside, it is valuable to understand the utility of ultrasound in diagnosing this disease. In this study, we compared ultrasound images of 7 neonates with their MR images obtained 524 hours later. We found that ultrasound was able to detect the lesions in 6 of the 7 patients, with the only false-negative ultrasound occurring in the patient with the smallest lesion. We also found that once the lesion was detected, ultrasound was able to separate and delineate the extra-axial pial hemorrhage and the underlying parenchymal abnormality, with their sizes and shapes correlating well with the MR imaging (Fig 4). To our knowledge, this is the first report on the value of cranial ultrasound in the diagnosis of this disease.

Third, we further analyzed the MR imaging features of the subpial hemorrhage and underlying infarct. We found that the underlying infarcted brain parenchyma often has concurrent hemorrhage, observed in 11 of 15 scans, which is similar to the reported $76 \%$ incidence of concurrent hemorrhage by Cain et $\mathrm{al}^{5}$ (Fig 2). In addition, we recognized the consistent combination of 

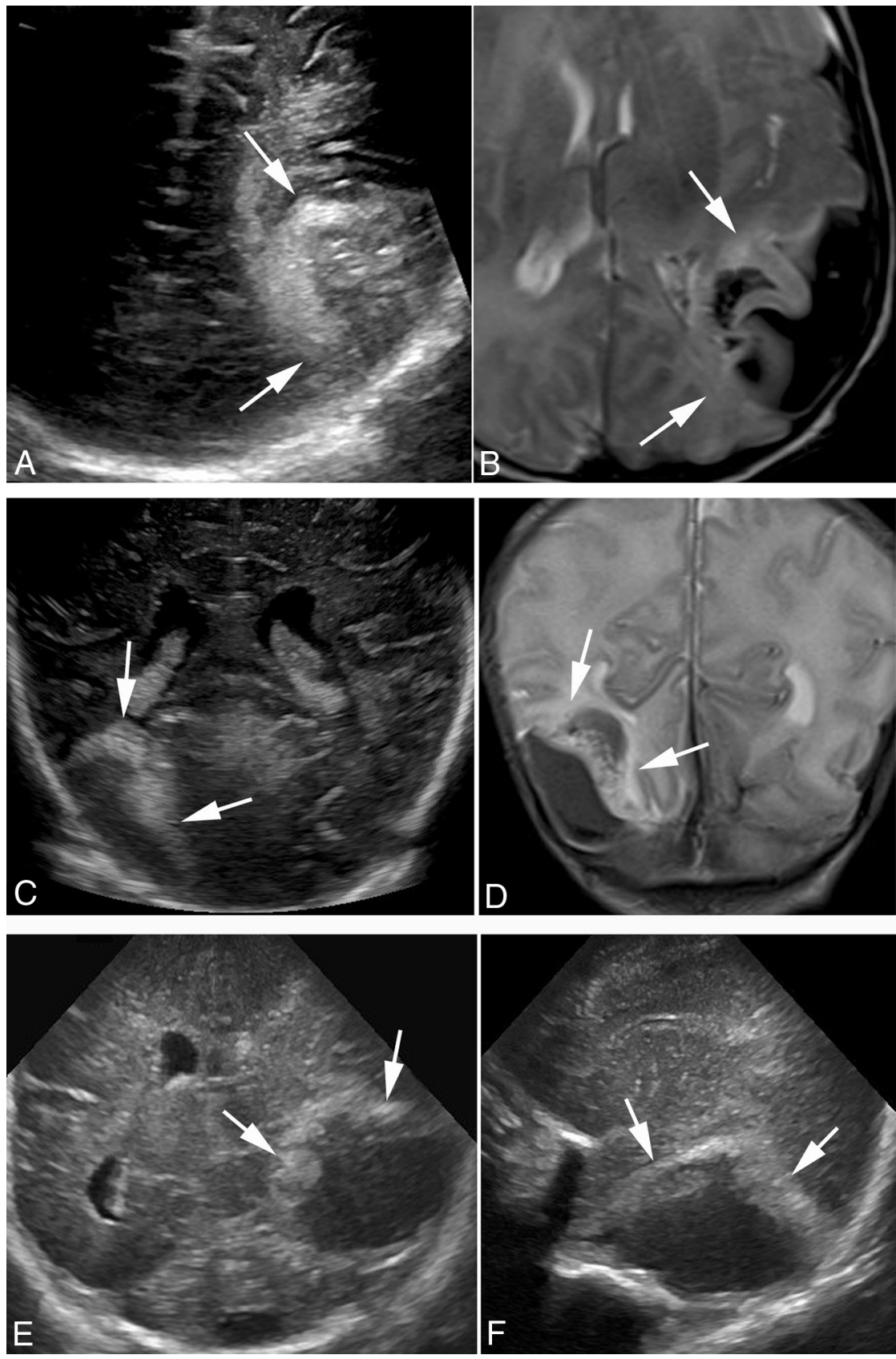

FIG 4. Ultrasound images of subpial hemorrhage with underlying cerebral infarct compared with MR images. The ultrasound images $(A$ and $C$ ) were obtained $<24$ hours before the MR images $(B$ and $D$ ) of a late-preterm neonate $(A$ and $B$ ) and a term neonate $(C$ and $D)$. Ultrasound is able to detect both the subpial hemorrhage and underlying cerebral infarct in both patients (arrows). The subpial collection of bleeds is mildly echogenic in the first patient $(A$ and $B)$ and hypoechoic in the second patient ( $C$ and $D$ ), even though they are all hypointense on T2-weighed MR images. Ultrasound is unable to differentiate the infarcted cerebral cortex from the underlying white matter with hemorrhagic infarct. The ultrasound images in the lower panel are from a very preterm neonate who did not have brain MR imaging performed before succumbing to disease.

brain parenchyma was seen in all 6 cases, which also gives a distinct imaging pattern that resembles the yinyang sign (Fig 4). Such a distinctive and memorable imaging biomarker may facilitate recognition by radiologists and clinicians.

Furthermore, we studied the natural course of the disease on serial MR images. On the follow-up MR images, neither the subpial hemorrhage nor the underlying infarct progressed with time. This observation may be useful clinically in guiding imaging followup of these patients.

Unfortunately, even though the imaging pattern of the subpial hemorrhage with underlying infarct described here and in previous reports ${ }^{4,5}$ is characteristic, the pathophysiology remains unknown. Subpial hemorrhages have been described using various terminologies in the literature, including leptomeningeal hemorrhage, superficial lobar hemorrhage, pial hemorrhage, extraaxial bleed with underlying infarct, and so forth. ${ }^{4,6,7}$ Many hypotheses have been proposed for the pathogenesis of this disease. Huang and Robertson ${ }^{4}$ suggested that local trauma with contusion or venous compression or occlusion may be the cause of the spontaneous superficial parenchymal and leptomeningeal hemorrhage in their cohort of term neonates. In our series, almost $70 \%$ of the infants were born by vaginal delivery. However, emerging evidence, including large case-controlled studies of neonatal hemorrhagic stroke, is confirming a lack of association with birth trauma. ${ }^{8}$ The study by Cain et $\mathrm{al}^{5}$ also concluded that a relationship with birth trauma was unlikely and that the imaging findings were instead suggestive of a nonarterial, deep venous pattern of hemorrhagic ischemia. Slaughter et $\mathrm{al}^{9}$ reported 7 term neonates with superficial temporal lobe hemorrhagic infarcts who presented immediately after birth to 14 days. Review of their MR images showed that at least 5 of their 7 cases

a bright, hyperintense appearance of the cerebral cortex with the dark, hypointense overlying subpial hemorrhage on T2WI and DWI, which creates a distinct imaging pattern that resembles the yin-yang symbol in Chinese philosophy (Fig 3). Similarly, on ultrasound, a consistent combination of a dark or grayish subpial collection of subpial bleeds with the underlying bright, echogenic had the yin-yang sign on T2WI. They attributed the findings to possible thrombosis of the vein of Labbe or other superficial temporal veins. However, they could not demonstrate a direct sign of venous thrombosis confidently on MRV. Neonatal cerebral sinovenous thrombosis is a well-described entity with similar imaging features; the inability of MRV to demonstrate venous thrombosis when the 
Table 4: Appearances of subpial hemorrhage and underlying brain parenchyma on follow-up MR imaging

\begin{tabular}{lclc}
\hline \multirow{2}{*}{$\begin{array}{c}\text { Time Interval between Initial } \\
\text { and Follow-Up MR Imaging }\end{array}$} & No. of Scans & \multicolumn{2}{c}{ Follow-Up MR (Appearances Compared with Initial MRI) } \\
\cline { 3 - 5 } 1-6 days & 8 & Subpial Hemorrhage & Underlying Cerebral Infarct \\
7-13 weeks & 4 & Evolved into fluid collection with less mass effect & Similar size and shape \\
13-28 weeks & 6 & Evolved into fluid collection with no mass effect & Decreased size \\
\end{tabular}

disease is limited to $\geq 1$ small cortical vein is well-known. ${ }^{10,11}$ While our retrospective, uncontrolled series cannot define the exact mechanisms, we would favor a similar mechanism as the most likely pathophysiology.

It also remains unclear whether it could be a cerebral venous infarct that causes secondary subpial hemorrhage; or instead, could a subpial hemorrhage cause venous compression and subsequent venous infarction of the underlying brain? The pia mater is a single-cell layered membrane closely adherent to the brain surface, only separated by a potential subpial space. The subpial space contains blood vessels and varying amounts of collagen. Arteries coursing through this space are always surrounded by the pia mater as they enter the parenchyma. However, the veins coursing through the subpial space may or may not be invested by the pia mater. ${ }^{12}$ Hence, it could be speculated that cortical veins, unlike cortical arteries, which have little-or-no leptomeningeal investment around them, can bleed directly into the subpial space. A certain amount of blood collection in the subpial space may potentially compress the underlying brain parenchyma, causing venous congestion, medullary vein thrombosis, and subsequent venous infarct. The presence of linear fan-shaped prominent deep medullary veins in some of our patients may represent deep medullary venous thrombosis, which would not be detectable on a MR venogram. On the other hand, while a strong network of trabeculae exists along veins and arteries in the subarachnoid space, this trabecular network is lacking around veins in the subpial space. This feature lends a potential mechanism for decompression of hemorrhage in superficial infarcts into the subpial space. ${ }^{13}$

Apnea and respiratory distress were the most presenting symptoms in our cohorts of patients (Table 1). These are not likely attributable to the direct effects of the hemorrhage itself (ie, compression). The proportion presenting with such clinical signs would seem comparable with other populations of neonates with acute, acquired focal brain injury. Whether this is due to seizures or other common respiratory reasons for neonates to be admitted to the neonatal intensive care units cannot be determined.

Our study is limited by its small sample size and its retrospective nature. The information on follow-up is limited and only available until a median of 18 months of age. A more thorough follow-up such as a Bayley scale assessment could not be realized in the current study design. As with all perinatal brain injury studies, true outcomes usually can only be determined with more comprehensive measures performed $>5$ years from birth. We hope further descriptions of the unique imaging findings will improve recognition and future studies to better understand pathophysiology and outcomes.

\section{CONCLUSIONS}

Subpial hemorrhage with underlying cerebral infarct is a condition that has a unique MR imaging and sonographic pattern that resembles the yin-yang symbol in Chinese philosophy. Ultrasound is able to detect all the subpial hemorrhages and underlying cerebral infarcts seen on MR imaging except for those that are relatively small. While this condition can occur in term and late-preterm neonates as previously reported, it can also occur in neonates born at extremely preterm or very preterm gestation. While the former group of patients has relatively mild neurologic sequelae, the latter group may have grave clinical outcomes. The pathophysiology of this condition is still not fully understood. We hope the further characterization of the imaging findings in our study will, to some degree, facilitate a better recognition in clinical practice and further research in the future.

\section{ACKNOWLEDGMENTS}

The authors would like to thank Amalia Floer for her administrative assistance.

Disclosures: Zarina Assis-UNRELATED: Employment: Radiologist at Alberta Children's Hospital, Calgary, Alberta, Canada. Adam Kirton-UNRELATED: Expert Testimony: occasional medical expert.

\section{REFERENCES}

1. Dunbar M, Kirton A. Perinatal stroke: mechanisms, management, and outcomes of early cerebrovascular brain injury. Lancet Child Adolesc Health 2018;2:666-76 CrossRef Medline

2. Dunbar M, Kirton A. Perinatal stroke. Semin Pediatr Neurol 2019;32:100767 CrossRef Medline

3. Lee S, Mirsky DM, Beslow LA, et al; International Paediatric Stroke Study Neuroimaging Consortium and the Paediatric Stroke Neuroimaging Consortium. Pathways for neuroimaging of neonatal stroke. Pediatr Neurol 2017;69:37-48 CrossRef Medline

4. Huang AH, Robertson RL. Spontaneous superficial parenchymal and leptomeningeal hemorrhage in term neonates. AJNR Am J Neuroradiol 2004;25:469-75 Medline

5. Cain DW, Dingman AL, Armstrong J, et al. Subpial hemorrhage of the neonate. Stroke 2020;51:315-18 CrossRef Medline

6. Bergman I, Bauer RE, Barmada MA, et al. Intracerebral hemorrhage in the full-term neonatal infant. Pediatrics 1985;75:488-96 Medline 
7. Hanigan WC, Powell FC, Palagallo G, et al. Lobar hemorrhages in fullterm neonates. Childs Nerv Syst 1995;11:276-80 CrossRef Medline

8. Cole L, Dewey D, Letourneau N, et al. Clinical characteristics, risk factors, and outcomes associated with neonatal hemorrhagic stroke: a population-based case-control study. JAMA Pediatr 2017;171:23038 CrossRef Medline

9. Slaughter L, Egelhoff J, Balmakund T. Neurologic outcome in neonatal temporal lobe hemorrhagic venous infarcts. J Child Neurol 2009;24:1236-42 CrossRef Medline

10. deVeber G, Andrew M, Adams C, et al; Canadian Pediatric Ischemic Stroke Study Group. Cerebral sinovenous thrombosis in children. N Engl J Med 2001;345:417-23 CrossRef Medline
11. Jordan LC, Rafay MF, Smith SE, et al; International Pediatric Stroke Study Group. Antithrombotic treatment in neonatal cerebral sinovenous thrombosis: results of the International Pediatric Stroke Study. J Pediatr 2010;156:704-10 CrossRef Medline

12. Weller RO, Sharp MM, Christodoulides M, et al. The meninges as barriers and facilitators for the movement of fluid, cells and pathogens related to the rodent and human CNS. Acta Neuropathol 2018;135:363-85 CrossRef Medline

13. Gunda D, Cornwell BO, Dahmoush HM, et al. Pediatric central nervous system imaging of nonaccidental trauma: beyond subdural hematomas. Radiographics 2019;39:213-28 CrossRef Medline 